\title{
INTESTINAL ANGULATIONS AND KINKS ASSOCIATED WITH STASIS.
}

\author{
BY W. I. DE C. WHEELER, F.R.C.S.I.; \\ Surgeon to Mercer's Hospital, Dublin.
}

[Read in the Section of Surgery, May 9, 1913.]

THE profusion of recent literature on the subject of intestinal stasis produced by non-inflammatory intestinal adhesions and kinks, with all the varying and contradictory expert opinions put forward, renders it difficult to compile a communication in any sense complete.

In this paper, for the sake of brevity, it will be necessary to confine my remarks to personal experiences with twenty recent abdominal cases which illustrated conditions associated with the term intestinal stasis. Some or all of the pathological conditions which I mention later in the paper were present in my series of cases.

It is not so long since we were taught that intestinal kinks and adhesions other than inflammatory were a product of the imagination - " a dagger of the mind, a false creation." Those who were first to discount the possibility of the presence of kinks and adhesions have now taken refuge in the statement that every individual has adhesions and kinks, and they do no harm. These two contentions are obviously impossible to adjust or harmonise.

It may be now taken as an axiom that in a large percentage of abdominal cases, cases of gastric or duodenal ulcer, appendicitis, gall-stones, and allied conditions, in- 
cluding cystic ovary, there is present a varying degree of intestinal stasis associated with certain pathological angulations and kinks to be mentioned later.

In a certain proportion of the cases these conditions, per se, give rise to no symptoms whatever; in others the symptoms are slight or masked; in the remainder they are so marked that a novice could not be deceived as to their cause.

The connection between, say, duodenal ulcer and intestinal kinks and other pelvic lesions is in dispute; that there is a close connection is beyond doubt, and has led Moynihan to remove the appendix in most cases of duodenal ulcer, and Lane to abandon the operation of gastroenterostomy for the relief of pyloric obstruction. From the admitted association of various intra-abdominal lesions there is one practical lesson to be learnt-the fallacy of performing very rapid operations for the removal of an appendix. If the appendix is in an anatomical position, free from pathological adhesions, and lends itself to rapid removal, either the operation is not justified or, more likely, the symptoms which called for the operation were due equally to other associated abnormal conditions. Surgery has been discredited by the persistence of symptoms after removal of an appendix by surgeons ignorant of the existence, say, of a Jackson's membrane or other abnormality. The presence of a surprisingly normal appendix in a patient who suffered from symptoms sufficiently severe to warrant operation should call for pause, and a careful search be made for abnormalities at the terminal part of the ileum, the ascending colon, and hepatic flexure, and if need be in all "four corners" of the abdominal cavity. The removal of an appendix in four or five minutes is easy of accomplishment, but does 
not savour of scientific surgery. The diagnosis of "appendix dyspepsia" associated with pyloric spasm in the light of recent advances in abdominal surgery can seldom be justified.

The following lesions were well marked in my series of cases, some in one, others in another; the presence of one abnormality did not necessarily mean the presence of another, although in several cases all the lesions to be mentioned were well marked and distinct in the same case. Thus, in some, appendicitis would be accompanied by a well marked Jackson's membrane, a Lane's kink near the ileo cæcal junction, and a mega-duodenum. In almost every case the terminal mesocolic kink was present, in some causing undoubted abdominal symptoms, in others doing no harm whatever.

Lane's terminal ileal kink was described as caused by a band forming part of the under surface of the mesentery and afterwards a distinct ligament which attached itself progressively to the ileum more and more distant from the mesenteric border. The band is shorter than the mesentery, thus causing a pucker or kink of the ileum, which is, in addition, often rotated on its long axis owing to the attachment of the band to the anti-mesenteric border. (Figs 1 and 2.)

According to Lane, this and other intestinal kinks are due to "the crystallisation of lines of force" to prevent prolapse of the viscera in cases of chronic constipation. Gray and Anderson do not accept the explanation, and point out that in both the lower ileal and lower sigmoid kinks the mesentery and mesosigmoid show no evidence of "the crystallisation of lines of strain" in the upper layers where most strain would be expected, and that in a number of cases of ileal kink there was no tendency 
whatever to a dropped cæcum, which, on the contrary, was well fixed. In my cases of ileal kink I could not satisfy myself that the small intestine was much, if at all, dilated above the lesion as described by Lane, but in two there was distinct evidence of mega-duodenum. This latter condition is produced not in any direct manner from the ileal kink, but by a similar adventitious formation at the duodeno-jejunal flexure, or to a band running across the duodenum just to the right of the superior mesenteric artery, as described by Murphy.

Jackson's membrane has attracted considerable surgical attention during the past twelve months. (Fig. 3.) It is a very obvious lesion found regularly in patients suffering from right-sided pain or discomfort with a loose, splashing cæcum, constipation, and some visceroptosis. It is present in other cases, but one soon gets familiar with a type of case where its presence can be foretold with almost certainty before operation. It is a thin, vascular veil, in which long, straight bloodvessels run a parallel course, compared by Jackson to an œdematous arachnoid. It is like a cobweb bag which is too small to hold the ascending colon without puckering it. It extends from the hepatic flexure to a variable extent over the colon on both sides of the anterior muscular band. It surrounds the caput of the cæcum, merges into the meso-appendix, and becomes lost in the pelvic peritoneum. Jackson's membrane is very frequently seen in children.

The better developed the Jackson's membrane, the more prolapsed and distended is the cæcum. This is an observation of importance, for it teaches that it does not assist in fixing a mobile cæcum, but, on the contrary, by sometimes producing a very acute kink at the hepatic 
flexure of the colon causes dilatation and ptosis of the cæcum and ascending colon. It is, therefore, more likely that Jackson's membrane is a cause, and not an effect, of intestinal stasis.

It is almost certain that Jackson's membrane is not inflammatory in origin. A study of the direction of the lymphatics proves it could not be a pericolic inflammation which had extended along the lymphatics of the subperitoneal coats. Furthermore, the blood-vessels run in a linear fashion, unlike those in inflammatory tissue, and little bleeding results from the general separation of the membrane. A sufficiently raw surface, however, in some instances requires the application of an omental graft to prevent subsequent adhesions.

Mayo's theory of abnormal burrowing of the cæcum and lower end of the ileum during their descent from the liver under the parietal posterior peritoneum has been dismissed owing to the absence of such a process in the examination of a very large number of fœtuses.

Gray and Anderson's is the most fascinating explanation, and appeals to the imagination of those who have studied the structures at operations. They say the membrane is really the right margin of the great omentum pulled out and thinned during development. The omentum crosses the hepatic flexure and blends by physiological fusion with the posterior parietal peritoneum to the outer edge of the colon. Fusion also takes place between the omentum and anterior wall of the ascending colon near the longitudinal muscular band in the same way as the omentum finds attachment to the transverse colon in a corresponding position. During the descent of the cæcum the omentum crossing the flexure and adherent to the parietal peritoneum externally gets pulled out, and is 
found in later life as the membrane already described. This theory is strengthened by the presence of fat, the linear arrangements of the vessels, and the site of attachment of the membrane to the colon. In several of my cases I could trace a direct and uninterrupted connection between the membrane and the omentum. This was specially noticeable above at the hepatic flexure, where Jackson's membrane often takes the form of powerful bands intermingled with fat, obstructing the onflow of the contents of the colon.

In addition to the ileal kink, the hepatic kink produced by Jackson's membrane, the dilated duodenum already referred to, I have had one case of Payr's disease, where there was a well marked kink of the splenic flexure, with dilatation and ptosis of the transverse and ascending colon, and collapse of the descending colon. It is a comparatively common condition, but I have only seen it twice before, once in a case operated upon by a colleague before its significance was understood, and once with Mr. Gray, of Aberdeen. The kink is probably due to a shortened phrenocolic ligament, or can be explained in precisely the same manner as the hepatic kink.

In two-thirds of my cases the lower sigmoid kink was present. So frequent is this condition seen that it is looked upon as almost normal by some authorities. All are agreed, however, that in certain cases it produces very marked constipation and left-sided pain with dilatation of the colon above. In many cases, on the other hand, it interferes with the bowel little, if at all. Here we can see a beautiful example of a kink produced by the excess of physiological fusion as described by Toldt.

Gray attributes the formation of all kinks to excess of physiological fusion, and it has been shown that the for- 
mation of Jackson's membrane can be attributed to a like cause. While Gray believes enteroptosis is produced by the presence of obstructive kinks, yet the congenital theory can be made clear by another interpretation.

When man assumes the erect position, in order to prevent a drop of all the viscera into the pelvis, nature, by physiological fusion of the visceral and parietal peritoneum, produces the splenic and hepatic flexures, the mesentery and meso-colon, and all ligaments intended to suspend the viscera.

Assume we approach at birth towards our ancestral condition, and suffer from slight congenital ptosis, or assume that later in life ptosis is produced from familiar causes, then there may be peritoneal fusion, in unusual places to ensure fixation as a provision of nature against further prolapse. To revert to the terminal kink of the pelvic colon, which is such a common condition, if we pull on the colon we can see the line of fusion between the outer side of the mesocolon and the peritoneum of the left iliac fossa. This line is known as Toldt's white line (Fig. 4.)

In cases of chronic constipation, in the absence of other causes, the " undoing" of this kink, especially in enteroptosis cases, will have the effect of producing a regular daily motion, and a consequent great improvement in the condition of this miserable class of patient.

I have operated upon several such cases for my colleagues, Drs. Lumsden and Holmes, with the result stated.

Left-sided abdominal pain with constipation may often be completely relieved by a plastic operation on this kink, and it should be remembered that the pain is often rendered more severe by implication of the left ovary and broad ligament in the line of fusion. (Figs. 1 and 5.) 
The congenital theory is not upset by the contention that symptoms do not arise until adult life. We have only to think of such conditions as cervical rib and congenital hernia to see where the fallacy of such a contention lies.

In two of my cases there were so many congenital adhesions and kinks that I short circuited the ileum into the lower portion of the pelvic colon-the operation recommended by Lane.

If the permanent results of the plastic work are as good as the immediate results the short circuiting operation will become unnecessary, but in any event the operation of ileosigmoidoscopy, as often attributed to Lane, is not performed in the manner he recommends, and gives correspondingly bad results. The ileum must be completely divided and anastomosed low down to the pelvic colon below the sigmoid kink.

If the kink is not well marked it should be artificially produced to prevent reverse peristalsis-a condition which, in early days, rendered necessary a subsequent colectomy in a fairly large proportion of cases. It is anatomically impossible to anastomose the ileum to the rectum as is often suggested.

In my series of cases the symptoms were very varied. Left-sided pain and discomfort by a process of elimination was diagnosed in several cases as being due to a well-developed mesosigmoid band with dilatation of the colon. These patients, usually females, were cured by operation.

Diffuse right-sided pain, tenderness, and discomfort in patients with a mobile splashing cæcum and distended ascending colon, were found usually to present a well marked Jackson's membrane and hepatic kink; the 
appendix often showed little or no signs of disease. The ileal kink was present in a few of these cases, but absent in at least an equal number. One case diagnosed as duodenal ulcer proved to be a megaduodenum accompanied by Jackson's membrane and ileal kink. The patient was a medical man, and presented all the signs and symptoms of duodenal ulcer. $X$-ray photographs supported this view, but on operation only the conditions mentioned were found. The patient was completely and immediately relieved by a plastic operation.

Two cases only were typical of auto-intoxication-there was staining and wrinkling of the skin, sweating in the axillæ and groins, enfeebled circulation with cold extremities, dulling of the mental faculties, \&c. The complete short circuit operation was performed. These patients are tolerably well. One was done two years ago, the other has just left hospital, but still suffers from abdominal distress. The number of ailments directly caused by intestinal stasis are probably legion, but of the remote lesions, such as chronic rheumatic arthritis, cystic breasts, and hyperthyroidism I have no personal knowledge, and, therefore, I only mention them to dismiss the subject.

$X$-ray photographs were taken of a number of the cases by Dr. Hayes to assist localisation of the lesion before operation.

I must acknowledge my great indebtedness to Sir Arbuthnot Lane and Mr. Gray for allowing me to see their cases and the results of operation. 
Mr. W. I. DE Courcy Wheeler on "Intestinal Angulations and Kinks Associated with Stasis."

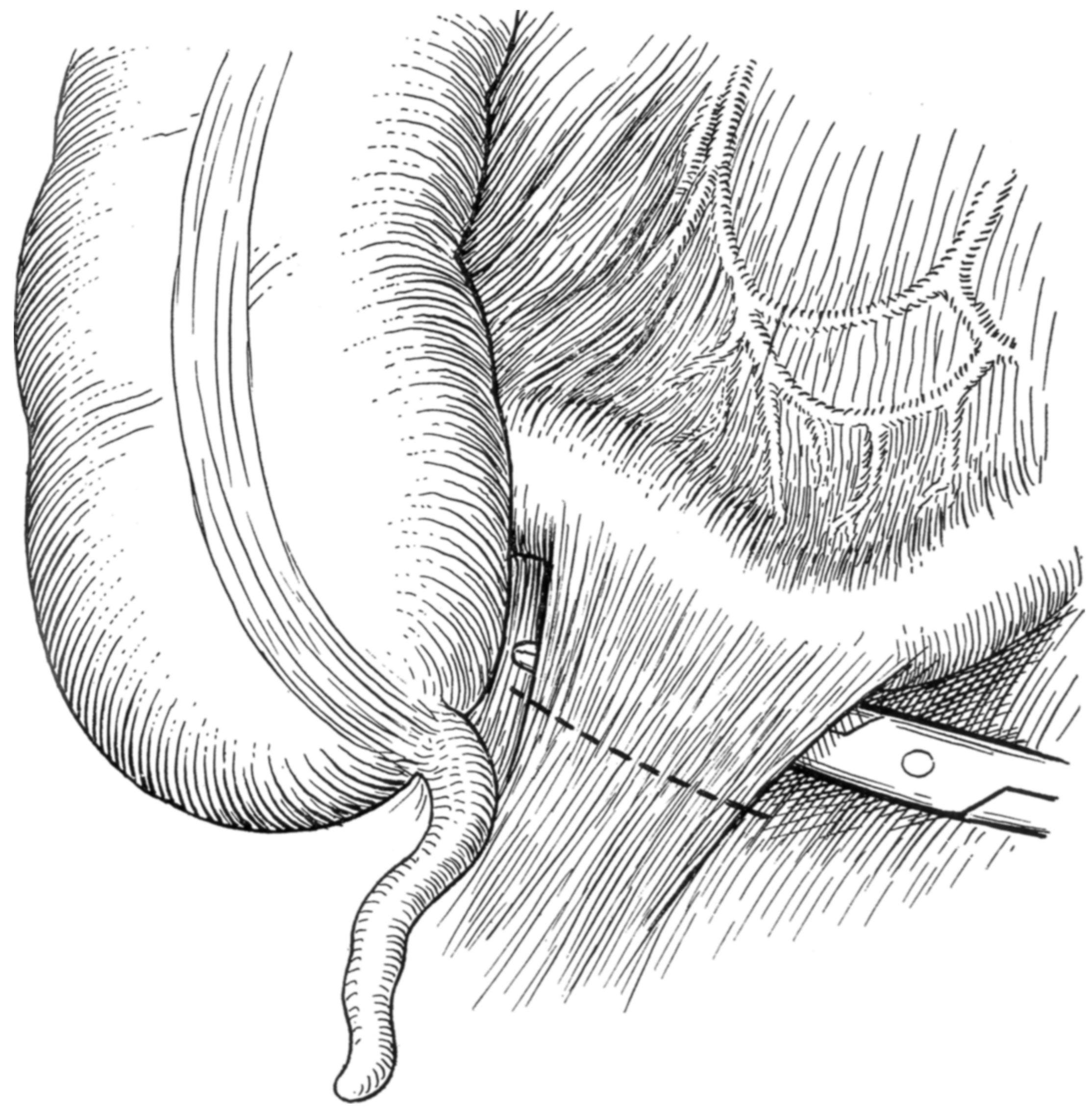

Fra. 1.-Diagram of Lane's terminal ileal kink, showing line of division in plastic operation. 
Mr. W. I. De Courcy Wheeler on "Intestinal Angulations and Kinks Associated with Stasis."

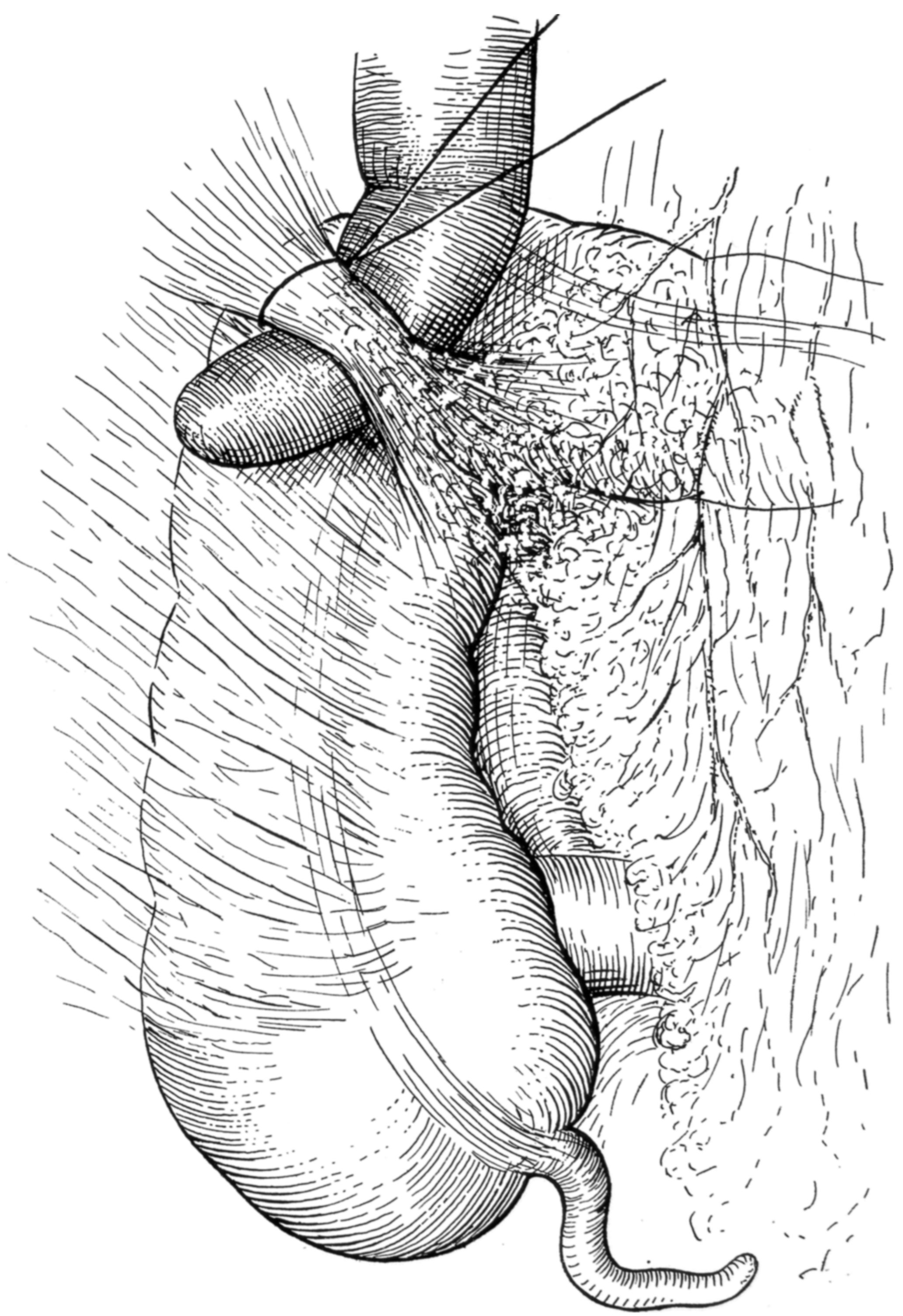

FIG. 3.-Jackson's membrane, showing the well developed band merging into the omentum obstructing the hepatic flexure and producing dilatation and ptosis of the cæcum. These cases are frequently referred to erroneously as "Appendix Dyspepsia." The bands are hooked up on the finger and divided from the flexure to the cæcum. 
Mr. W. I. de Colrcy Wheeler on "Intestinal Angulations and Kinks Associated with Stasis."

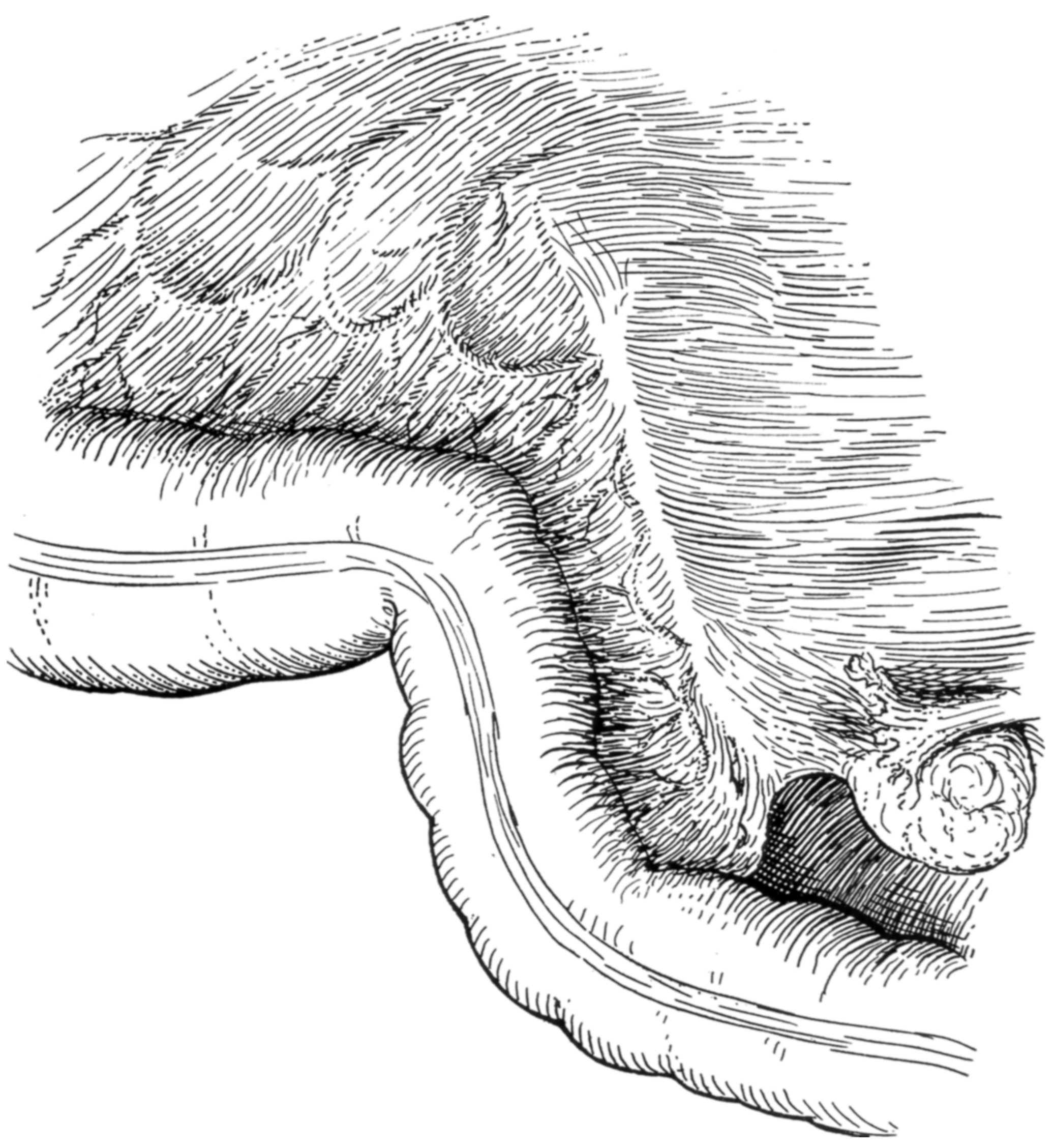

FIG. 4.-.-Kink of the pelvic colon showing "Toldt's Line" and implication of the left ovary. Obscure left-sided pain and constipation are frequently produced by this condition and relieved by operation. (Modified from GRay aNd ANDERSON.) 
Mr. W. I. De Courcy Wheeler on "Intestinal Angulations and Kinks Associated with Stasis."

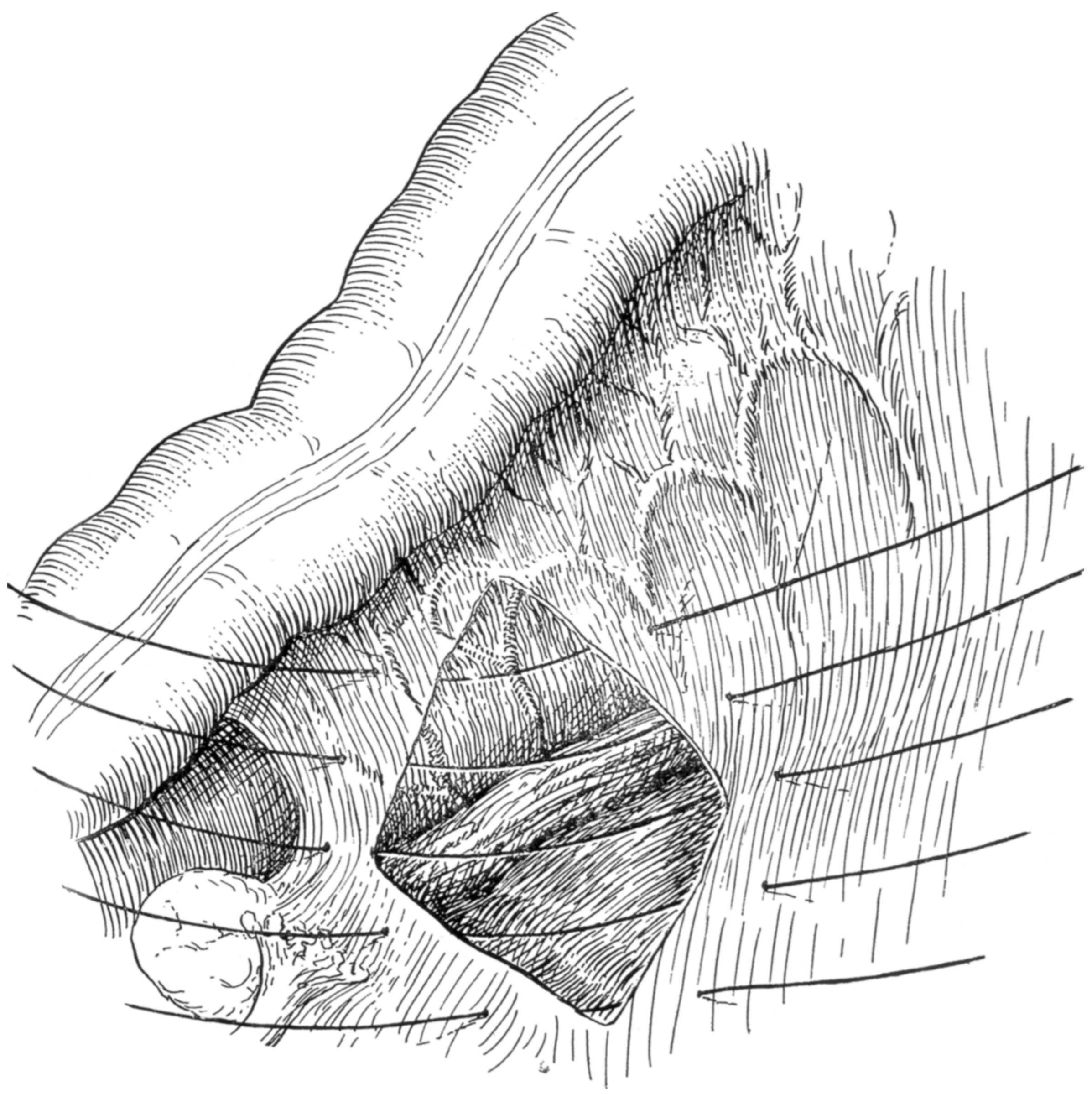

FI(T. 5.---Kink of the pelvic colon relieved by plastic operation. (Modified from Gray aNd ANDerson.) 